\title{
Special issue on multidimensional systems applications
}

\author{
Paula Rocha ${ }^{1}$. Youqing Wang ${ }^{2}$
}

Published online: 26 August 2015

C) Springer Science+Business Media New York 2015

Although originally motivated by applications such as image processing, multidimensional $(\mathrm{nD})$ systems have mainly attracted the attention of the research community due to the richness of new theoretical questions raised by their treatment, and are often replaced by other settings when it comes to modelling, identifying and controlling processes in practical situations.

The aim of this special issue is to illustrate the application potential of $\mathrm{nD}$ systems by presenting several contributions where the $\mathrm{nD}$ framework is successfully used either to deal with practical problems or to solve practically relevant questions that arise in other contexts that are not at first sight related to multidimensional systems.

In the paper Multidimensional Control Systems: Case Studies in Design and Evaluation, by Rogers et al., after introducing the most commonly used models for the analysis and control of discrete $\mathrm{nD}$ linear systems, a series of case studies is presented with the aim of demonstrating the advantages of the $\mathrm{nD}$ systems approach in solving certain control problems. Namely, applications in the fields of sensor grid networks, iterative learning control, civil engineering and agriculture are considered.

The paper Two-dimensional Generalized Predictive Control (2D-GPC) Scheme for the Batch Processes with Two-dimensional (2D) Dynamics, Shi et al. studies the problem of iterative learning control (ILC) design for batch processes. Based on 2D controlled auto-regressive moving average (2D-CARMA) model, a novel two-dimensional generalized predictive control (2D-GPC) is proposed. The feasibility and effectiveness of the proposed method is validated by the experimental results on packing pressure control of the injection molding process.

Paula Rocha

mprocha@fe.up.pt

Youqing Wang

wang.youqing@ieee.org

1 Research Center for Systems and Technologies (SYSTEC), Faculdade de Engenharia da Universidade do Porto, Rua Dr. Roberto Frias, 4200-465 Porto, Portugal

2 College of Information Science and Technology, Beijing University of Chemical Technology, Beijing 100029, People's Republic of China 
As is well-known, repetitive processes can be viewed as a special class of $\mathrm{nD}$ processes. Azevedo-Perdicoúlis et al. present the paper Modelling a Gas Pipeline as a Repetitive Process: controllability, observability and stability, where criteria for these structural properties are derived considering that the system is steered by initial conditions and boundary data. These results are relevant for the management of high pressure gas networks.

The paper Decentralized String-Stability Analysys for Heterogeneous Cascades Subject to Load-Matching Requirements, by Soltanian and Cantoni, studies the stability of heterogeneous cascades of stable LTI subsystems in terms of the spatial and temporal propagation of boundary conditions. A decentralized string-stability certificate is established in order to validate certain requirements on the desired behavior, which allows a local controller design in order to achieve a string-stable behavior overall. This is applied to the design of distributed distant dowstream controllers for the sections of an automated irrigation channel.

Internally passive multidimensional Kirchhoff circuits have been successfully used to model the 2D shallow water equations, which play an important role in the fluid dynamics literature. Basu and Fettweis have made great contributions in this field and discuss here yet a new method in their paper A New Look at 2D Shallow Water Equations of Fluid Dynamics via Multidimensional Kirchhoff Paradigm.

In the paper Failure Identification for Linear Repetitive Processes, Maleki et al. investigate the fault detection and isolation (FDI) problem for discrete-time linear repetitive process using a geometric approach in 2-D system framework. Sufficient conditions for solvability of this problem are given. Furthermore, a statistical approach for determining a decision threshold has been developed when there exists noise.

Zhao et al. consider the problem of sensor fault reconstruction and compensation for a class of 2-D nonlinear systems in their paper entitled Sensor Fault Reconstruction for A Class of 2-D Nonlinear Systems with Application to Fault Compensation. The considered sensor fault can be of arbitrary form and its size can be even unbounded. An integrated fault/sensor observer is proposed to obtain the asymptotic estimation of sensor faults and system states simultaneously, for which a sufficient existing condition is given and proven.

The use of dynamic neural networks has proved to yield good results in parameter estimation for 1D systems. Based on these results, in the paper A 2D Hopfield Neural Network Approach to Mechanical Beam Damage Detection, Almeida et al. propose a 2D version of a Hopfield Neural Network that can be used for damage detection in vibrating beams by monitoring significant changes in the estimated beam parameters. The good performance of this method is illustrated by simulations.

The paper $n D$ Methods for 1D Parameter-Dependent Systems, by Zerz and Walcher, illustrates the use of $\mathrm{nD}$ system theoretic tools for the analysis of other kinds of systems. In particular, the authors show how certain Gröbner basis mehods, originally developed for $\mathrm{nD}$ systems, may be effective in the study of issues as controllability and controlled invariant varieties for polynomial parameter-dependent systems. Moreover they show how nD methods can also be used in the study of certain aspects of nonlinear parameter dependent state space systems and illustrate this with an application to the study of the dynamics of an infectious disease.

We would like to thank all the authors for their contributions, the reviewers for their valuable suggestions, and, last but not least, the Editor-in-Chief, Zhiping Lin, for his constant help and support. 


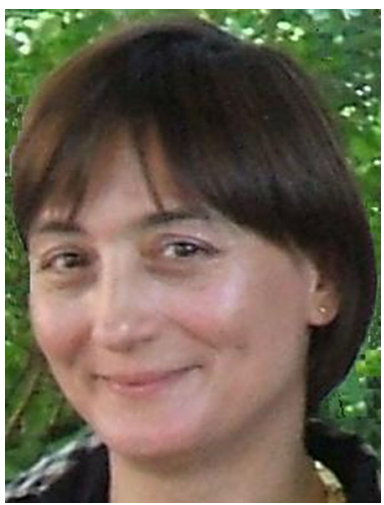

Paula Rocha graduated in Applied Mathematics at the University of Porto and obtained her Ph.D. degree in Systems and Control from the University of Groningen, The Netherlands. After having worked as an Assistant Professor at the Department of Technical Mathematics of the Delft University of Technology, she moved to the Department of Mathematics of the University of Aveiro, Portugal, where she has been a Professor till the end of 2008. She is currently a Professor at the Department of Electrical and Computer Engineering of the Faculty of Engineering, University of Oporto, Portugal. Her interests are mainly in the area of Systems and Control, namely in the framework of the behavioral approach and in the field of multi-dimensional (nD) systems, as well as in applications to biomedical systems.

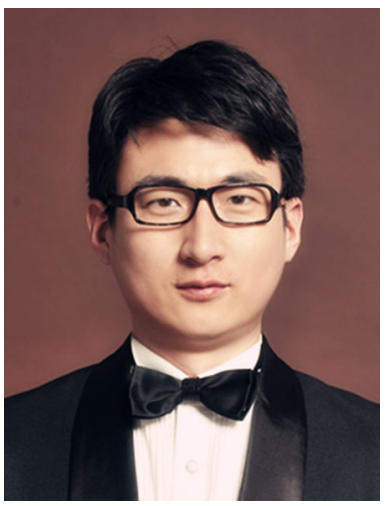

Youqing Wang received his B.S. degree from Shandong University, Jinan, Shandong, China, in 2003, and received his Ph.D. degree in control science and engineering from Tsinghua University, Beijing, China, in 2008. He worked as a Research Assistant in the Department of Chemical Engineering, Hong Kong University of Science and Technology, from February 2006 to August 2007. From February 2008 to February 2010, he worked as a Senior Investigator in the Department of Chemical Engineering, University of California, Santa Barbara, CA, USA. From March 2008 to December 2011, he was an Adjunct Senior Investigator in Sansum Diabetes Research Institute. From August 2015 to November 2015, he was a Visiting Professor in University of Alberta. Since 2010, he is a Full Professor in Beijing University of Chemical Technology. His research interests include faulttolerant control, state monitoring, modeling and control of biomedical processes (e.g., artificial pancreas system), and iterative learning control. Prof. Wang is an Associate Editor of Multidimensional Systems and Signal Processing. He is a member of IFAC Technical Committee on Chemical Process Control and IFAC Technical Committee on Biological and Medical Systems. He is the recipient of several research awards (including Journal of Process Control Survey Paper Prize, ADCHEM Young Author Prize, and IEEE CSS Beijing Chapter Young Author Prize). 